\title{
Recent Advances of STATCOM in Power Transmission Lines - A Review
}

\section{Mr. L NarayanaGadupudi ${ }^{a}$, Dr.GudapatiSambasivaRao ${ }^{b}$}

${ }^{2}$ Research Scholar, Dept. of EEE, AcharyaNagarjuna University, Guntur, Andhra Pradhesh.,glngitam@gmail.com

${ }^{\mathbf{b}}$ Professor, Dept. of EEE, R.V.R. \& J.C. College of Engineering, Guntur, A.Andhra Pradhesh.sambasiva.gudapati@gmail.com

Article History: Received: 10 November 2020; Revised 12 January 2021 Accepted: 27 January 2021; Published online: 5 April 2021

\begin{abstract}
Internal Liability of power system transmission lines influenced by the turbulences owing to catastrophic disasters. In order to achieve Constant Voltage Stability at both ends of the transmission lines, Static Synchronous Compensator (STATCOM) is imperative. Voltage source Converter mechanisms augment with switching frequency control methodologies are widely adopted to regulate the reactive power. By deliberating IEEE Standards, the minimization of Total Harmonic Distortion (THD) is conceivable with STATCOM. This paper depicts the advancement of VSC based STATCOM approaches and the methodologies to minimize the switching losses. Economical management of High-Power ratings systems is also discussed in this paper.
\end{abstract}

Keywords:

\section{Introduction}

Electrical Power generation is a significant component to estimate the growth of a nation. Inadequate electrical power at various regions and limited power generation sources is alarming a country growth. The Voltage disturbance, impedance disturbance, and differences in phase angle are impacting to save the power both at transmission and at receiving end. Reactive power regulation and subsequently, constant system voltage maintenance, in order to minimize the losses is challenging today. Minimize the running expenses for long duration is significant. A typical STATCOM model circuit is given below in Fig.1

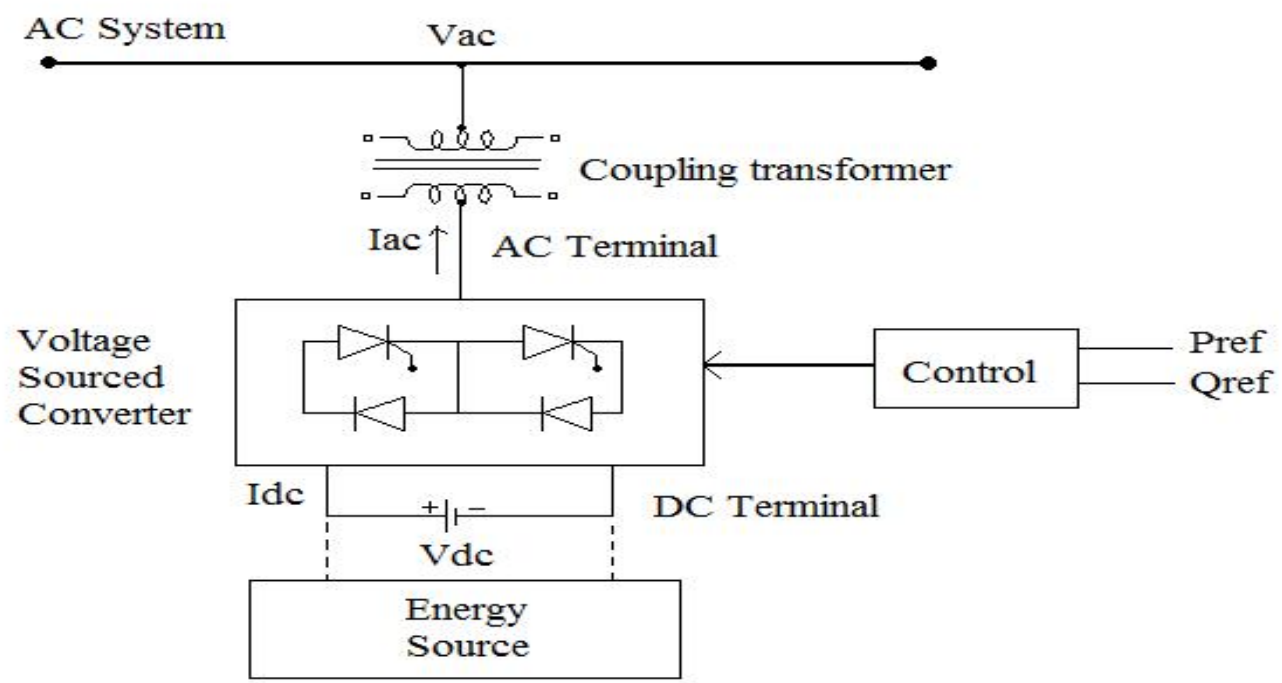

Fig.1 STATCOM circuit model

Enhancement of system utilization and power quality is essential by adopting the STATCOM in association with power electronics-based FACTS device. For Power transmission line demand, flying capacitor, H-Bridge circuits and Multi-level multi pulse diode clamped (VSC Methodologies) are essential. For Improving the abrupt response speed and bi-directional active and reactive power flow capability, VSC based STATCOM Control 
methodologies augment with PI, Fuzzy logic, artificial intelligence, And FPGA is deliberated. In order to achieve continuous voltage control to keep power system voltage stability and to curtail the losses in power system, STATCOM is imperative.

\section{Analysis of STATCOM:}

The Power Losses disintegration, regulating the Current and adequate DC voltage balance is attainable with multi-level STATCOM methodologies. Cascade H-Bridge (CHB) based configuration is an appropriate choice to address the above stated characteristics. Linear and Non-Linear Control approaches and even anticipatory (Predictive) Control Approach are significant for Controlling the current precisely. For mapping the system with reference frame then add to $\mathrm{P}$ plus I Controller tuned with either root-locus and bode diagram, linear control approaches is compelling.

Since the compensating current is not depending on voltage level, In STATCOM, parallel converter algorithm based on capacitor voltage is significant. Specifically, Reactive Power support in wind farms is influenced with SATCOM in order to release power flow bottleneck and support voltage. The quantity of wind turbines estimate based on the actual voltage level required and estimated demand in network [1]. The comprehensive performance of the system is impacted by the election of a control methodology. The elected controller modeling and dynamic response influences on generation of firing signal to power electronic switches. In Wind Farms, the control methodology of the STATCOM is persuaded by the compensating signal. To enhance the system dynamics then for VAR Compensation, shunt connected compensator, i.e., Static condenser static Synchronous compensator is endorsed. With SVC the reliable response is under privileged. VSC with fast Fourier Series or with pulse width modulation switching scheme improves the fast and reliable response. The dynamic performance is elevated with VSC multi-level and multi pulse STATCOM methodologies. Though, GTO-VSC based STATCOM approach is more reliable, for enhancing the benefits, multi-level inverter approach (IGBT) is elected as reliable approach. The capacity of power transfer at transmission and at receiving stage determines the Structure of STATCOM. These characteristics motivating to improve the structure of STATCOM include VSC methodology [2]. DC capacitor voltage will determine the VSC output voltage for STATCOM operation. The phase angle difference of converter as well as system voltages will determined by dc link voltage for improvement of reactive power in the system

A novel methodology is proposed a '2' groups used for ' 3 ' level 24-pulse VSCs by fixed dc voltage aimed at STATCOM operation of reactive power regulating. Also, the phase angle has been estimated with nominal variation of system as well as converter voltages [3]. Modular Multi level E-STATCOM is proposed a Photovoltaic Energy storage to exchange the power for utility grides. It provides real as well as reactive powers and harmonic filtering. The MMC based configuration is capable to handle higher power over conventional two or three level inverter-based topologies due to modularity, low losses, better harmonic profile, etc. The constant power is infusing into the grid with E-STATCOM for compensating the varying power produced by PV and MPPT Operation. For improved grid stability and to cut down the fluctuations of PV power MMC based ESTATCOM is recommended, which in turn as PV Dominated Grid [4]. The efficiency is significantly improved over '2' or ' 3 ' level E-STATCOM. This approach is more compelling for High power applications. In STATCOM for dynamic reactive power compensation, for reliable voltage stabilization, and for dynamic power flow control in electrical networks, gate turnoff thyristor control augment with VSC is more appropriate [5]. Regulate the bus voltage, while aiding the reactive power demand, STATCOM is a reliable approach. The transmission line utilization is significantly improved with this approach [6]. This approach is greatly enhancing the dynamic stability, active power balance and reactive power compensation issues. Transformer coupling, and application of reactor and capacitor for compensation is laborious and cumbersome. The same performance can be harvested with multilevel inverters ostracize the transformer coupling [7].

STATCOM is likely mechanism for promoting full bridge sub- module (a small repeating unit) for HVDC. For STATCOM MMC converters and for HVDC the sub model is a significant building block. With full-bridge submodule and GE'S Grid solutions are able to provide complete harmonic free determinations and discourses the entire exact requirements [8]. The STATCOM has the following operation characteristics of

- $\quad$ Fast response to provide reactive power and hence manage voltage disturbances.

- Small footprint.

- Low maintenance.

- Relocatability.

- Good harmonic performance.

- Low noise generation.

- Low associated magnetic fields. 


\section{Statcom methodologies:}

\section{i. Cascade H-Bridge:}

The Conditional Inductive operation is impeding the application of H-bridge STATCOM (LC-STATCOM) with minimum capacitor value. Because of Minimum Capacitance value, the complete inductive operation is not able to cover with LC-STATCOM. An extra reactor value is added to the STATCOM in order to defend the limitation with LC-STATCOM. Large inductor size is elected for parallel compensator. Significantly small inductor size is elected for series compensator. In Inductive region, The LC-STATCOM is proposed to compensate the overall operating range. For Grid Voltage regulation of inductive and capacitive operating regions, both parallel and series compensation LC-STATCOM adds upgraded decisive performance [9]. The delta Hbridges based STATCOM is controlled the reactive current moving in a system and unbalancing loads by dc-link voltage [10]. For Dynamic performance regulation, upper and lower limit values of dc capacitor voltages for LCSTATCOM is deliberated. The upper and limit values curtail the strain on power electronic devices. This is achieved with LC-STATCOM over other conventional methodologies [11]. The converter losses are managed by maintain the constant dc-link voltage for reactive power improvement in multi cascade VSC based STATCOM. Voltage source is used for output module voltage representation and the angular frequency of source voltage is used to achieve the voltage equation at steady state [12].

\section{ii. Diode-clamped MMC (DCMMC):}

In each sub module of DCMMC, a small diode power range is enough for clamp the capacitor voltage. Two simple methodologies are innovated such as voltage balanced control as well as constant capacitor voltage control. The response speed is significantly high for capacitor voltage balance control. VAR Compensation and negative sequence current compensation is harvested with modular multilevel converter-based DCM STATCOM. This methodology minimizes the number of capacitor voltage sensors to a significant value. And fast balancing control is quite easy over other methods. Though the number of clamping diodes and inductors are used, but Lower current rating of diode and inductors are elected over conventional MMC methodologies [13]. In order to maintain constant dc capacitor voltage, three phase MMC based STATCOM is proposed with series connected single phase modules. The System Voltage peak values and constant dc capacitor voltages is meticulously estimated by adopting control algorithms for proposed STATCOM. For medium voltage ac distribution system applications, the size miniaturization is dominated (eliminating the bulky line frequency transformers) with MMC mechanisms, which are reliable to promote direct connected converters [14].

\section{iii. CSC based STATCOM:}

For medium voltage ac drive motors, input current quality and load voltages maintenance, CSC (Current source Converters) based STATCOM is recommended. The load disturbances in drives are able to regulate with CSC based STATCOM instead normal voltage converters. Employing CSC for active power filters is limiting owing to power losses with DC Current. This can be eclipsed current level compensation operations in STATCOM [15].

\section{iv. Double bridge series converter:}

Recommending re-injection transformer may transform multilevel re-injection circuit into voltage source converter and also as double bridge series converter. The Converter system voltage output pulses are significantly improved and subsequently total hormonic distortion is drop-off considerably. This mechanism is recommended for STATCOM applications [16]. For Enhancing the Performance of the system, circuit breakers are reliable choice to isolate ac and dc grid connections in HVDC Grid structures. Addition of static synchronous compensators may further elevate the efficiency in transmission system [17].

\section{v. Fuzzy PI controller STATCOM:}

STATCOM with Fuzzy-P+I mechanism for SEIG (Self excited induction generator) feed load balance and unbalance conditions is a recommended choice for voltage regulation in Transmission systems. The capacitor voltage is influencing on STATCOM performance. During stat up significantly high dc Voltage across the capacitor is observed with this methodology. The Fuzzy P+I voltage scheme regulates the dc capacitor voltage results the performance is improved [18]. Recommending STATCOM for GTO and Voltage Source Converter may produce 48-Pulse. Three leg VSC and phase shifting transformer is backing for this converter. This approach is generating or absorbing equivalent flow of current to rated reactive power compensation and exchange the reactive power with Ac System. The current harmonics are significantly eradicating with this approach. The disturbances in Ac system are able to be governed with STATCOM [19]. 


\section{vi. Hybrid communication STATCOM topology:}

In Cascade H- Bridge STATCOM with Master and Slave Control configuration, distributed control is adopted with Hybrid Communication STATCOM. Which will minimize the maintenance overheads. The H-Bridge Modules are interconnected with slave controllers driven by master controllers for enhancing the system efficiency in closed loop structure. The Communication loops are operating in parallel at different layers of three phase modules [20]. Control system de-coupling for cascading H-Bridge Multi level Converter is an added advantage with STATCOM. In traditional STATCOM based cascade H-bridge m-level converters, the dc-link voltage levels are balanced with individual voltage controllers. Owing to coupling effect, the cluster voltage output as well as current output controllers are amended [21]. For wind power controlling, energy storage is a competent mechanism. Cascaded multi-level STATCOM is the base for Hybrid energy storage system which will smoothen the Wind power variations. A Super capacitor and a Battery are essential for this mechanism. A control strategy is to be employed for active and reactive power coordination [22].

\section{vii. Direct control STATCOM:}

Reactive power supervision with multilevel STATCOM and total hormonic regulation is achieved by adopting Level shift sine pulse width modulation. The Voltage is significantly dissolved and constant dc voltage is maintained under load unbalance conditions with Direct current control STATCOM approach [23]. Four distinguished methodologies have proved for current control cascaded H-Bridge STATCOM labeled as 1. linear strategy using PI controllers in the dq frame, 2. an exact input/output linearization technique with proportionalresonant controller, 3. A multi-band hysteresis modulation control and, 4.Predictive control scheme. The merits and demerits of each methodology is depicted with reference to stationary and dynamic performance, harmonic content, complexity, and computational overhead [24]. For Large power ratings, multi-pulse and multilevel VSC based STATCOM is recommended. The cost is significantly increased with increasing the pulse number and levels of the system. Fundamental frequency switching is base for three level 6 pulse diode clamped GTO-VSC STATCOM which is more recommended for high power applications [25].

\section{viii. Predictive and Phase shift STATCOM control:}

For multi-level H-Bridge Converters, PCM (Phase shift carrier modulation) is recommended industry standard. At low carrier frequencies required in high power applications is susceptible to hormonic deterioration. Space vector Index augment with level shifting modulation will enhance the system performance [26]. The reactive power and voltage harmonics are regulated with derived predictive control equations. Model predictive control mechanism used for CHB based STATCOM to improve the system performance. STATCOM operation is evaluated with controlling as well as controlled values to derive appropriate equations for model based predictive control. STATCOM as well as predictive control outputs difference signal is estimated by feedback correction as well as rolling optimization controllers for system smooth operation [27].

\section{ix. De capacitor balance based STATCOM:}

HBMLI (Cascade H-Bridge multi-level inverters) are proposed for applications like reactive power compensation, solid state transformers and pv systems. The unbalancing of high-level converters and dc capacitor voltages are administrated with the proposed methodology. Negotiating the dc link unbalance voltage may be seriously damage the semiconductor components. CHBMLI (dc link Capacitor voltage balancing methodology) will surpass the limitation. Fast voltage balancing is achieved with this approach [28]. In order to improve the grid performance at varying load conditions, difference of dc capacitor voltage is estimated periodically. So that, the estimation of switching losses and active power losses is made easy to improve the performance. The grid performance at varying unbalance conditions is further enhanced by deliberating the Zero sequence control and negative sequence control methodologies [29].

In Cascade H- Bridge, Capacitor voltage ripple impacts on current quality. A Static Compensator with low capacitance value is deliberated for equivalent I-V Characteristics. Different capacitor ripple voltages estimation is imperative for inductive and capacitive operating modes of $\mathrm{H}$ - Bridge Converter. Also, THD of converter output voltage is considered [30]. To Curtail the strain on the semiconductor devices and to safe guard from failures, MBPC (Model based Predictive Control) is developed. Which is labeled as E-S i.e., energy storage integrated STATCOM. Controlling the reactive power in a H-Bridge STATCOM will improve the system performance. MMC is turned into STATCOM and energy storage is built within Dc link of MMC [31]. ESTATCOM is basically operated as STACOM and able to support active power at various instances of time. This methodology will address the PV Related conflicts and act as PV dominated Grid [32]. 


\section{x. Asymmetrical MLC STATCOM:}

The reactive power is compensated by adopting the 27-level symmetric multi- level Master slave compensator. The dc voltage is able to regulate with master PI Control loop. Proportional resonant control block in the inner loop maintains the reference currents [33].

\section{Conclusion}

This paper projects the detailed VSC based STATCOM with different power circuits and various control circuits. It can be concluded that, if the power rating of the transmission lines rises then the switches count will increase. But the power quality of the system will be deteriorating because the power electronic switches loss will increase with switches count. So, switching losses is inversely proportional to the system power quality. Actually, over a period of time if power rating increases neither the switches nor the switching losses will be change. On observing the over view, it is identified that, as switching losses decreases then the system efficiency increases. These leads to new techniques of adoptive control strategies with novel topologies.

\section{References}

I. Sanz, M. Moranchel, E. J. Bueno, F. J. Rodriguez, “Analysis of medium voltage modular multilevel converters for FACTS applications" 42nd Annual Conference of the IEEE Industrial Electronics Society, 23-26 Oct. 2016.

N.G.Hingorani, L.Gyugi, understanding FACTS: "Concepts and Technology of flexible AC transmission systems”, IEEE, New York, 2000.ISBN 0-7803-3455-8.

Bhim Singh, VenkataSrinivasKadagala "Simulation of three level 24-pulse voltage source converters based static synchronous compensator for reactive power control" IEEE Trans. On power electronics, ISSN 1755-4535, Oct 2013.

Anil Bharadwaj, SumanMaiti "Modular multilevel E-STATCOM considering distributed energy storage at the dc link" IEEE Power India International Conference, 25-27 Nov. 2016.

SubhasisBandopadhyay "Digital Simulation of 72-Pulse GTO based STATCOM for Reduced Harmonic Injection to Grid”, IEEE Smart Energy Grid Engineering (SEGE), 21-24 Aug 2016.

Gaurav Kumar, ParagNijhawan "Simulation-Based performance analysis of three-level 48-pulse STATCOM with constant DC link voltage for reactive power compensation" 7th India International Conference on Power Electronics, 17-19 Nov 2016.

Omkar K. Shinde, VRSV BharathPulavarthi, Sagar S. Patil "Five level inverter based STATCOM with PI control" International Conference on Circuit, Power and Computing Technologies, 20-21 April 2017.

Javier Chivite-Zabalza, Jerome Perrier, Mike Boden, TianningXu, John Outram "Development of a full-bridge sub-module for HVDC and STATCOM markets" International Exhibition and Conference for Power Electronics, Intelligent Motion, Renewable Energy and Energy Management, 16-18 May 2017.

Glen Farivar, Christopher David Townsend, BranislavHredzak, JosepPou, Vassilios G. Agelidis "Passive Reactor compensated cascaded H-bridge multilevel LC-STATCOM”, IEEE Trans. on Power Electronics, Volume: 32, Issue: 11, pp. 8338 - 8348, Nov 2017.

Jae-Jung Jung, Joon-Hee Lee, Seung-Ki Sul, Gum Tae Son and Yong-Ho Chung "DC Capacitor Voltage Balancing Control for Delta-Connected Cascaded H-Bridge STATCOM Considering the Unbalanced Grid and Load Conditions", IEEE Trans. on Power Electronics, Vol: 33, Issu: 6, pp. 4726-4735, June 2018.

GhiasFarivar, Christopher David Townsend, BranislavHredzak, JosepPou and Vassilios G. Agelidis "LowCapacitance Cascaded H-Bridge Multilevel StatCom", IEEE Trans. on Power Electronics, Volume: 32, Issu: 3, pp. 1744 - 1754, March 2017.

Yu Sugahara, TakaharuTakeshita "Reactive Current Control of a Multi-DC-Voltage Cascade STATCOM", International Conference on Renewable Energy Research and Applications (ICRERA), 22-25 Nov 2015.

Xiangdong Liu, JingliangLv, CongzheGao, Zhen Chen, Si Chen "A Novel STATCOM based on diode clamped modular multilevel converters", IEEE Transactions on Power Electronics, Volume: 32, Issue: 8, pp. 5964 5977, Aug 2017.

TakanoriIsobe, Long Zhang, Ryuji Iijima, Hiroshi Tadano, Yasuhiko Kawanami and Katsushi Terazono "Experimental Verification of capacitance reduction in MMC based STATCOM", IEEE Energy Conversion Congress and Exposition, 18-22 Sep. 2016.

Pedro E. Melín, Benjamin Larenas, Jeovanny Valenzuela, Jaime A. Rohten, Javier A. Riedemann, M. Nathalie Risso "Multilevel Current-Source Converter based STATCOM using Minimum DC Current Level" International Conference on Automatica, 19-21 Oct. 2016. 
K. R. Patel and S. M. Hosseini "Performance Analysis of reinjection circuit in multi-pulse Voltage Source Converter for STATCOM applications", IET International Conference on Power Electronics, Machines and Drives, 19-21 Apr. 2016.

Abel Antonio-Ferreira, OriolGomisBellmunt, MiquelTeixido "HVDC-based modular multilevel converter in the STATCOM operation mode", European Conference on Power Electronics and Applications, 5-9 Sep. 2016.

JyotirmayeeDalei, KanungoBaradaMohanty, Sumit Singh, Gour Sunder Garain "Fuzzy PI controller for improved voltage regulation in STATCOM based SEIG", Annual IEEE India Conference (INDICON), 17-20 Dec. 2015.

AbderrahmenAbdellaoui, AbderrazakYangui, Amara Saidi, HsanHadjAbdallah "STATCOM based 48-Pulses Three Level GTO Dedicated to VAR Compensation and Power Quality Improvement", International Conference on Sustainable Mobility Applications, Renewables and Technology, 23-25 Nov 2015.

HuaGeng, Shuzhen Li, Chao Zhang, Geng Yang, Lei Dong, BabakNahid-Mobarakeh“ Hybrid Communication Topology and Protocol for Distributed-Controlled Cascade H-Bridge Multilevel STATCOM”, IEEE Trans. on Industry Applications, Volume: 53, Issue: 1, pp. 576 - 584, Jan. 2017.

GhiasFarivar, BranislavHredzak, Vassilios G. Agelidis "Decoupled Control System for Cascaded H Bridge Multilevel Converter based STATCOM”, IEEE Trans. on Industrial Electronics, Volume: 63, Issue: 1, pp. 322 - 331, Jan 2016.

Han Yashuai, XuYonghai and Li Yangfan "Research on Application of STATCOM/HESS in Wind Power Integrated to the Grid", IEEE International Future Energy Electronics Conference, 1- 4 Nov. 2015.

S.A. Kamran, Javier Munoz "Study of a state-of-the art M-STATCOM", IEEE International Conference on Industrial Technology, 17-19 Mar. 2015.

Javier Munoz, Jaime Rohten, Jose Espinoza, Pedro Melin, Carlos Baier and Marco Rivera “ Review of Current Control Techniques for a Cascaded H-Bridge STATCOM", IEEE International Conference on Industrial Technology, 17-19 Mar. 2015.

RadheshyamSaha and Bhim Singh "A 3X6 Pulse NPC-VSC based 100 MVAr STATCOM Modeling and Applications”, IEEE Power India International Conference, 5 - 7 Dec 2014.

Christopher D. Townsend, Roberto A. Baraciarte, Yifan Yu, Daniel Tormo, Hector Zelaya de La Parra, Georgios D. Demetriades, Vassilios G. Agelidis "Heuristic Model Predictive Modulation for High-Power Cascaded Multi-Level Converters", IEEE Transactions on Industrial Electronics, Volu: 63, Issue: 8, pp. 5263 - 5275 , Aug 2016.

XuRong, Yu Yong, Wu Jian, Yang Rongfeng, XuDianguo, Chen He, Yu Yannan and Ni Ronggang "Improved Model Predictive Control for H-bridge Cascaded STATCOM", International Conference on Power Electronics and ECCE Asia, 1-5 Jun 2015.

Jalal Amini and MehrdadMoallem "A Capacitor Voltage Balancing Method for Cascaded H-Bridge Multilevel Inverters with Application to FACTS”, Annual Conference of the IEEE Industrial Electronics Society, 23-26 Oct 2016.

EhsanBehrouzian and Massimo Bongiorno "Impact of capacitor balancing strategies on converter ratings for star-connected cascaded H-bridge STATCOM", European Conference on Power Electronics and Applications, 11-14 Sep 2017.

Glen Farivar, JosepPou, AnshumanTripathi "LC-StatCom with symmetrical I-V characteristic, Total Harmonic Distortion Study", Asian Conference on Energy, Power and Transportation Electrification, 24-26 Oct 2017.

L.Comparatore, J. Rodas, M. Rivera, R. Gregor, J. Pacher, A. Renault, J. Muñoz and P. Sanjeevikumar "Model Based Predictive Control with a Fixed Switching Frequency applied to a Single-Phase Cascade H-Bridge Multilevel STATCOM", IEEE Conference on Industrial Electronics and Applications, 18-20 Jun 2017.

Anil Bharadwaj and SumanMaiti "Modular Multilevel STATCOM with Energy Storage", International Conference on Industrial and Information Systems, 3-4 Dec 2016.

Javier Munoz, PatricioGaisse, FaustoCadena, Carlos Baier, Rodrigo Aliaga, Jose Troncoso "Proportional Resonant Controller for a 27-Level Asymmetric Multilevel STATCOM", Chilean Conference on Electrical, Electronics Engineering, Information and Communication Tech, 18-20 Oct 2017. 